\title{
A Dengue Vaccination Model for Immigrants in a Two-Age-Class Population
}

\author{
Hengki Tasman, ${ }^{1}$ Asep K. Supriatna, ${ }^{2}$ \\ Nuning Nuraini, ${ }^{3}$ and Edy Soewono ${ }^{3}$ \\ ${ }^{1}$ Department of Mathematics, Universitas Indonesia, Depok 16424, Indonesia \\ ${ }^{2}$ Department of Mathematics, Universitas Padjadjaran, Jatinangor 45363, Indonesia \\ ${ }^{3}$ Department of Mathematics, Institut Teknologi Bandung, Bandung 40132, Indonesia
}

Correspondence should be addressed to Hengki Tasman, htasman@sci.ui.ac.id

Received 18 October 2011; Revised 2 January 2012; Accepted 17 February 2012

Academic Editor: A. Zayed

Copyright (C) 2012 Hengki Tasman et al. This is an open access article distributed under the Creative Commons Attribution License, which permits unrestricted use, distribution, and reproduction in any medium, provided the original work is properly cited.

\begin{abstract}
We develop a model of dengue transmission with some vaccination programs for immigrants. We classify the host population into child and adult classes, in regards to age structure, and into susceptible, infected and recovered compartments, in regards to disease status. Since migration plays important role in disease transmission, we include immigration and emigration factors into the model which are distributed in each compartment. Meanwhile, the vector population is divided into susceptible, exposed, and infectious compartments. In the case when there is no incoming infected immigrant, we obtain the basic reproduction ratio as a threshold parameter for existence and stability of disease-free and endemic equilibria. Meanwhile, in the case when there are some incoming infected immigrants, we obtain only endemic equilibrium. This indicates that screening for the immigrants is important to ensure the effectiveness of the disease control.
\end{abstract}

\section{Introduction}

Dengue fever is an endemic disease in many tropical countries, especially in the urban areas. This disease is caused by the dengue virus, which is transmitted to a human by the bite of infected female Aedes aegypti mosquitoes.

There are some epidemiological and demographical factors that contribute to the transmission of the disease. Age factor is among the important demographical factors affecting the transmission of the disease. From a theoretical point of view, age structure affects the dynamics of the disease transmission [1], and hence it should be taken into account in modeling the transmission of the disease to increase the realism of the model and to obtain a more prudent decision derived from the model. From a practical point of view, many 
vaccination programs are directed to a certain class of age, unexceptionally in the case of dengue in which the Pediatric Dengue Vaccine Initiative targets children in their vaccination program (http://www.pdvi.org/). A study in [2] shows that a pediatric vaccination would be economically viable and highly cost effective, once a perfect dengue vaccine is made. A similar study shows that an optimal vaccination strategy could be given to only certain classes of age [3].

In literatures, most of the age-structured population models appear in the form of integropartial differential equations [4-6]. Some authors included age structure in epidemic models in the form of discrete compartmental differential equations, such as in [7-9]. The authors in [7] have generalized the model in [10] by separating the human population into age cohorts, and then for each cohort they construct a set of SIR equations. Diseasefree and endemic equilibria are found, but there is no stability analysis for these equilibria. In [8], the authors have simplified their model to a two-age-class model. They allowed different transmission rates for the adult and the child classes and found disease-free and endemic equilibria. They also provided the condition for the local stability of the disease-free equilibrium in the general case. The stability condition for the endemic equilibrium has only been found for the special case, in which no infection occurs for the adult class.

The authors in [9] showed that a two-age-class model is a special case of a more general continuous age model for a certain choice of survival function. In their paper they discussed a two-age-class dengue transmission model by dividing the human population into child and adult classes and considered vaccination in the child class only. Many scientists believe that most dengue infections are asymptomatic. For every ten cases we see in the hospital, there should be at least 50-90 cases in the community who have only fever and no complications [11]. In this regards, the authors in [9] also showed that, in some circumstances, if there is an inadvertent vaccination to asymptomatic infectious children, which worsens their condition as the time span of being infectious increases, then paradoxically, vaccination can be counterproductive; that is, vaccination makes the basic reproduction number even bigger. This suggests that, in practice, screening to identify truly susceptibles is needed before implementing a vaccination program.

Beside age factor, another factor that plays important role in disease transmission is immigration. It is easy to understand that immigration of infectious individuals could ignite the spreading of a disease in a virgin populations. Diseases like HIV, SARS, and avian influenza are believed among the examples of diseases that might be caused by the immigrants of infectious individuals $[12,13]$. Many mathematical models have been devised as the means to understand and to control those kinds of diseases $[6,14,15]$. The authors in [14] showed that if there is a constant influx of infective immigrants into a population, there will be no disease-free equilibrium.

Although the immigrants are not carrying a disease at all, still they have an impact on the transmission of a disease. The buildup of immigrants (also the locals) can be viewed as the buildup of susceptibles that are ready to be infected by any disease once available or enhance the spreading of the existing disease. In this respect, it is reasonable to enforce a policy to vaccinate incoming immigrant, following a screening, to ensure that they will not contribute to the buildup of susceptible.

There is no commercially dengue vaccine available yet. However, there are some potential dengue vaccines available. A survey in four South-Eastern Asian countries in 2002 revealed that there is a high and urgent perceived need for a dengue vaccine (http://www .pdvi.org/). To simulate vaccination program in gaining some insight on how vaccination would affect the transmission of the disease, even before the vaccine itself is available in 
the market, is among the interests of vaccine scientists and policy makers. In this paper we develop a two-age-class model for dengue transmission by considering immigration vaccination strategy, as an anticipative study before the vaccine exists.

The introduction of immigration into the system is plausible since dengue is regarded as an urban disease [16], where the rate of immigration cannot be neglected. Different from [9] in which it is assumed that vaccination targets individuals in the child class, here we look at a scenario where vaccination is given to a portion of newborns (both immigrant and local babies) and a portion of newly arrived mature immigrants, to protect them from being infected by the local dengue disease. In practical point of view, the vaccination strategy proposed in this paper is easier to be implemented than the one in [9].

\section{Model Formulation}

Let us assume that the host population is classified into the child class and the adult class. Each of the classes is divided into the susceptible, infected, and recovered subclasses. We also assume that the recovered hosts have life-long immunity and there is no wanning effect of the vaccine, which means that the vaccine has a life-long permanent protection. So, the recovered hosts and the vaccinated hosts can be grouped into the recovered class.

We use variables $\widetilde{S}_{C}, \widetilde{I}_{C}$, and $\widetilde{R}_{C}$ to denote the size of the susceptible, infected, and recovered of child population, respectively. Similarly, we use the subscript $A$ for the adult population.

We denote the susceptible, exposed, and infected vector populations by $S_{V}, E_{V}$, and $I_{V}$, respectively. We consider the latent class $E_{V}$, since the incubation period of the disease in mosquitoes is relatively large compared to the life span of the mosquitoes.

We use the diagram in Figure 1 for the dengue transmission in the population. The parameters $P_{C}$ and $P_{A}$ are the incoming immigration recruitment rates for child and adult classes, respectively, some positive fractions $f_{*}, g_{*}$, and $h_{*}$ of the incoming immigrants are susceptible, infected, and recovered or vaccinated, respectively $\left(f_{*}+g_{*}+h_{*}=1\right)$. In practice, it is necessary to undertake screening to identify the susceptibility status of the incoming immigrants. There is also a constant birth recruitment rate $B$ that increases the child population.

The parameters $p$ and $q$ are the fractions of susceptible incoming children (including natural birth) and susceptible incoming adults that are vaccinated; $s$ is the vaccine efficacy; $\mu_{C}, \mu_{A}$, and $\mu_{V}$ are the child, adult, and vector natural death rates; respectively, $\varepsilon_{C}$ and $\varepsilon_{A}$ are the per capita emigration rates for children and adults, respectively; $\lambda_{C}, \lambda_{A}$, and $\lambda_{V}$ are the successful infection rates for children, adults, and vectors; respectively, $\delta$ is the transition rate from child class to adult class; $\gamma$ is the recovery rate, $P_{V}$ and $1 / \tau$ are the recruitment rate for vector and the latent period of vectors, respectively.

Using the transmission diagram in Figure 1, we formulate the following 9-dimensional model:

$$
\begin{gathered}
\frac{d \widetilde{S}_{C}}{d t}=(1-p s)\left(f_{C} P_{C}+B\right)-\lambda_{C} \frac{\widetilde{S}_{C}}{\widetilde{N}_{H}} \widetilde{I}_{V}-\left(\delta+\varepsilon_{C}+\mu_{C}\right) \tilde{S}_{C} \\
\frac{d \tilde{I}_{C}}{d t}=g_{C} P_{C}+\lambda_{C} \frac{\widetilde{S}_{C}}{\widetilde{N}_{H}} \widetilde{I}_{V}-\left(\delta+\gamma+\varepsilon_{C}+\mu_{C}\right) \tilde{I}_{C}
\end{gathered}
$$




$$
\begin{aligned}
& \frac{d \tilde{R}_{C}}{d t}=h_{C} P_{C}+p s\left(f_{C} P_{C}+B\right)+\gamma \tilde{I}_{C}-\left(\delta+\varepsilon_{C}+\mu_{C}\right) \tilde{R}_{C}, \\
& \frac{d \widetilde{S}_{A}}{d t}=(1-q s) f_{A} P_{A}+\delta \widetilde{S}_{C}-\lambda_{A} \frac{\widetilde{S}_{A}}{\widetilde{N}_{H}} \widetilde{I}_{V}-\left(\varepsilon_{A}+\mu_{A}\right) \widetilde{S}_{A}, \\
& \frac{d \tilde{I}_{A}}{d t}=g_{A} P_{A}+\lambda_{A} \frac{\widetilde{S}_{A}}{\widetilde{N}_{H}} \widetilde{I}_{V}+\delta \tilde{I}_{C}-\left(\gamma+\varepsilon_{A}+\mu_{A}\right) \tilde{I}_{A}, \\
& \frac{d \tilde{R}_{A}}{d t}=\left(h_{A}+q s f_{A}\right) P_{A}+\delta \tilde{R}_{C}+\gamma \tilde{I}_{A}-\left(\varepsilon_{A}+\mu_{A}\right) \tilde{R}_{A}, \\
& \frac{d \widetilde{S}_{V}}{d t}=P_{V}-\lambda_{V} \widetilde{S}_{V} \frac{\widetilde{I}_{C}+\widetilde{I}_{A}}{\widetilde{N}_{H}}-\mu_{V} \widetilde{S}_{V} \\
& \frac{d \tilde{E}_{V}}{d t}=\lambda_{V} \widetilde{S}_{V} \frac{\tilde{I}_{C}+\tilde{I}_{A}}{\widetilde{N}_{H}}-\left(\tau+\mu_{V}\right) \widetilde{E}_{V} \\
& \frac{d \tilde{I}_{V}}{d t}=\tau \widetilde{E}_{V}-\mu_{V} \tilde{I}_{V}
\end{aligned}
$$

where $\widetilde{N}_{H}$ is the total population of host. Furthermore, we use $\widetilde{N}_{C}=\widetilde{S}_{C}+\widetilde{I}_{C}+\widetilde{R}_{C}, \widetilde{N}_{A}=$ $\widetilde{S}_{A}+\widetilde{I}_{A}+\widetilde{R}_{A}$, and $\widetilde{N}_{V}=\widetilde{S}_{V}+\widetilde{E}_{V}+\widetilde{I}_{V}$ as the total populations of child, adult, and vector, respectively. These populations are governed by the following equations:

$$
\begin{gathered}
\frac{d \widetilde{N}_{C}}{d t}=P_{C}+B-\left(\delta+\varepsilon_{C}+\mu_{C}\right) \widetilde{N}_{C} \\
\frac{d \widetilde{N}_{A}}{d t}=P_{A}+\delta \widetilde{N}_{C}-\left(\varepsilon_{A}+\mu_{A}\right) \widetilde{N}_{A}, \\
\frac{d \widetilde{N}_{V}}{d t}=P_{V}-\mu_{V} \widetilde{N}_{V} .
\end{gathered}
$$

When $t \rightarrow \infty$, we have that $\widetilde{N}_{C} \rightarrow\left(P_{C}+B\right) /\left(\delta+\varepsilon_{C}+\mu_{C}\right), \widetilde{N}_{A} \rightarrow\left(\delta\left(P_{C}+B\right)+\left(\delta+\varepsilon_{C}+\right.\right.$ $\left.\left.\mu_{C}\right) P_{A}\right) /\left(\delta+\varepsilon_{C}+\mu_{C}\right)\left(\varepsilon_{A}+\mu_{A}\right)$, and $\widetilde{N}_{V} \rightarrow P_{V} / \mu_{V}$.

First, we consider that the host and vector populations have reached the limiting states; these are $\widetilde{N}_{C}=\left(P_{C}+B\right) /\left(\delta+\varepsilon_{C}+\mu_{C}\right), \widetilde{N}_{A}=\left(\delta\left(P_{C}+B\right)+\left(\delta+\varepsilon_{C}+\mu_{C}\right) P_{A}\right) /\left(\delta+\varepsilon_{C}+\mu_{C}\right)\left(\varepsilon_{A}+\right.$ $\left.\mu_{A}\right), \widetilde{N}_{V}=P_{V} / \mu_{V}$, and $\widetilde{N}_{H}=\widetilde{N}_{C}+\widetilde{N}_{A}$. Then, we scale model (2.1)-(2.9) with following transformations $S_{C}=\widetilde{S}_{C} / \widetilde{N}_{C}, I_{C}=\widetilde{I}_{C} / \widetilde{N}_{C}, R_{C}=\widetilde{R}_{C} / \widetilde{N}_{C}, S_{A}=\widetilde{S}_{A} / \widetilde{N}_{A}, I_{A}=\widetilde{I}_{A} / \widetilde{N}_{A}$, $R_{A}=\widetilde{R}_{A} / \widetilde{N}_{A}, S_{V}=\widetilde{S}_{V} / \widetilde{N}_{V}, E_{V}=\widetilde{E}_{V} / \widetilde{N}_{V}$, and $I_{V}=\widetilde{I}_{V} / \widetilde{N}_{V}$. Thus, we obtain the following reduced model:

$$
\begin{gathered}
\frac{d S_{C}}{d t}=(1-p s)\left(f_{C} Q_{C}+T\right)-\beta_{C} S_{C} I_{V}-\left(\delta+\varepsilon_{C}+\mu_{C}\right) S_{C}, \\
\frac{d I_{C}}{d t}=g_{C} Q_{C}+\beta_{C} S_{C} I_{V}-\left(\delta+\gamma+\varepsilon_{C}+\mu_{C}\right) I_{C},
\end{gathered}
$$




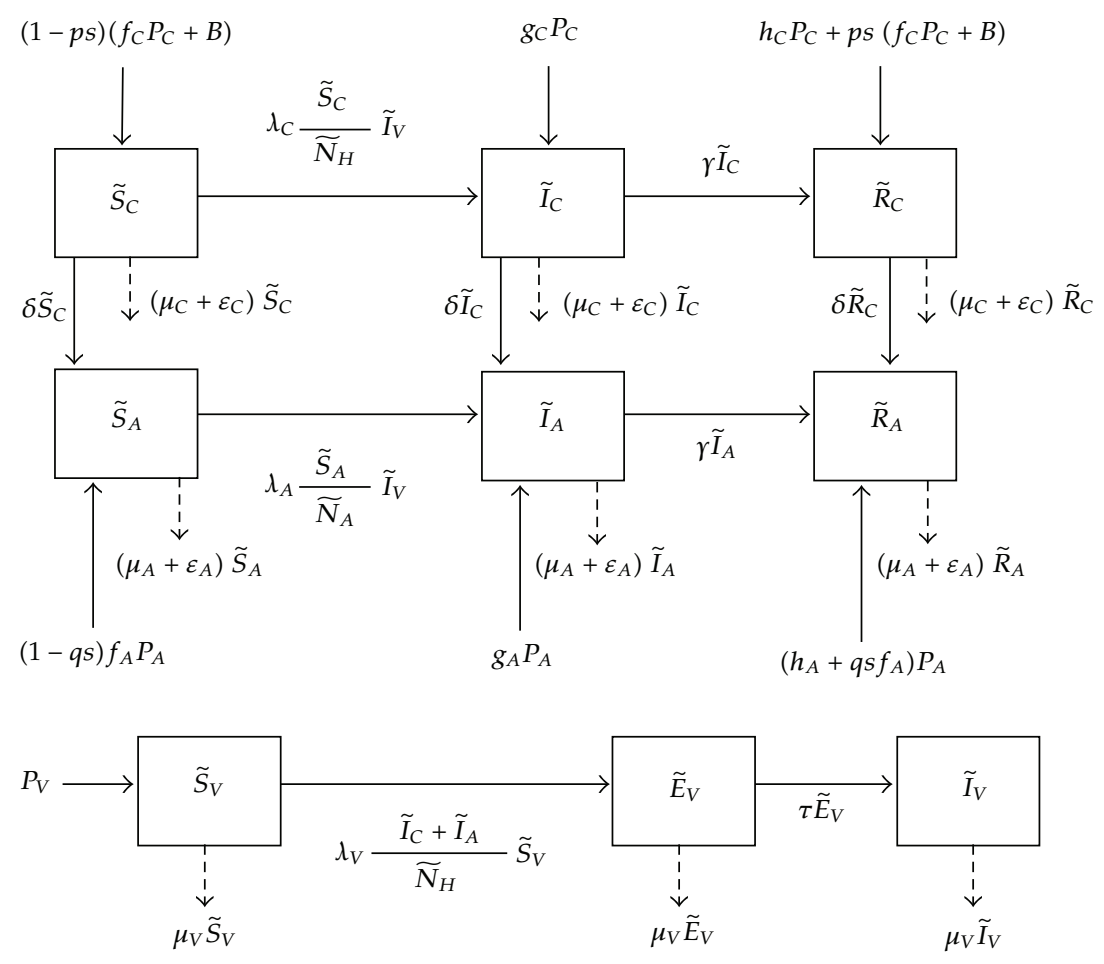

Figure 1: A two-age-class dengue transmission diagram.

$$
\begin{gathered}
\frac{d S_{A}}{d t}=(1-q s) f_{A} Q_{A}+\delta \sigma S_{C}-\beta_{A} S_{A} I_{V}-\left(\varepsilon_{A}+\mu_{A}\right) S_{A}, \\
\frac{d I_{A}}{d t}=g_{A} Q_{A}+\beta_{A} S_{A} I_{V}+\delta \sigma I_{C}-\left(\gamma+\varepsilon_{A}+\mu_{A}\right) I_{A}, \\
\frac{d E_{V}}{d t}=S_{V}\left(\theta_{C} I_{C}+\theta_{A} I_{A}\right)-\left(\tau+\mu_{V}\right) E_{V}, \\
\frac{d I_{V}}{d t}=\tau E_{V}-\mu_{V} I_{V}
\end{gathered}
$$

where $T=B / \widetilde{N}_{C}, Q_{C}=P_{C} / \widetilde{N}_{C}, \beta_{C}=\lambda_{C} \widetilde{N}_{V} / \widetilde{N}_{H}, \theta_{C}=\lambda_{V} \widetilde{N}_{C} / \widetilde{N}_{H}, \sigma=\widetilde{N}_{C} / \widetilde{N}_{A}, Q_{A}=$ $P_{A} / \widetilde{N}_{A}, \beta_{A}=\lambda_{A} \widetilde{N}_{V} / \widetilde{N}_{H}, \theta_{A}=\lambda_{V} \widetilde{N}_{A} / \widetilde{N}_{H}$, and $S_{V}=1-E_{V}-I_{V}$. The values of $R_{C}$ and $R_{A}$ in the limiting state can be evaluated using $R_{C}=1-S_{C}-I_{C}$ and $R_{A}=1-S_{A}-I_{A}$.

After the scaling, the region of biological interest of model (2.13)-(2.18) is

$$
\Omega=\left\{\left(S_{C}, I_{C}, S_{A}, I_{A}, E_{V}, I_{V}\right) \in[0,1]^{6}: S_{C}+I_{C} \leq 1, S_{A}+I_{A} \leq 1, E_{V}+I_{V} \leq 1\right\} .
$$

This region is positive invariant under the flow generated by the vector field of model (2.13)(2.18), because the vector field on the boundary of $\Omega$ does not point out the exterior of $\Omega$. 
For the rest of the paper, we will analyze model (2.13)-(2.18) since this reduced model is the limiting system of model (2.1)-(2.9) and has the same asymptotic behavior as the original model $[17,18]$.

\section{Model Analysis}

Solving the equilibrium conditions of model (2.13)-(2.18), we obtain the following equations:

$$
\begin{gathered}
S_{C}=\frac{(1-p s)\left(f_{C} Q_{C}+T\right)}{\beta_{C} I_{V}+\delta+\varepsilon_{C}+\mu_{C}}, \\
I_{C}=\frac{g_{C} Q_{C}+\beta_{C} S_{C} I_{V}}{\delta+\gamma+\varepsilon_{C}+\mu_{C}}, \\
S_{A}=\frac{(1-q s) f_{A} Q_{A}+\delta \sigma S_{C}}{\beta_{A} I_{V}+\varepsilon_{A}+\mu_{A}}, \\
I_{A}=\frac{g_{A} Q_{A}+\beta_{A} S_{A} I_{V}+\delta \sigma I_{C}}{\gamma+\varepsilon_{A}+\mu_{A}}, \\
E_{V}=\frac{\mu_{V}}{\tau} I_{V},
\end{gathered}
$$

and the variable $I_{V}$ satisfies $M\left(I_{V}\right)+N\left(I_{V}\right)=0$, where

$$
\begin{aligned}
M\left(I_{V}\right)= & \left(c_{2} I_{V}^{2}+c_{1} I_{V}+c_{0}\right) I_{V} \\
N\left(I_{V}\right)= & \left(I_{V} \beta_{A}+\varepsilon_{A}+\mu_{A}\right)\left(I_{V} \beta_{C}+\delta+\varepsilon_{C}+\mu_{C}\right)\left(I_{V}\left(\mu_{V}+\tau\right)-\tau\right) \\
& \times\left[g_{A} Q_{A} \theta_{A}\left(\gamma+\delta+\varepsilon_{C}+\mu_{C}\right)+g_{C} Q_{C}\left(\theta_{C}\left(\gamma+\varepsilon_{A}+\mu_{A}\right)+\theta_{A} \delta \sigma\right)\right] \\
c_{2}= & \beta_{A} \beta_{C}\left(\mu_{V}+\tau\right)\left[(1-q S) f_{A} Q_{A} \theta_{A}\left(\gamma+\delta+\varepsilon_{C}+\mu_{C}\right)\right. \\
& \quad+\mu_{V}\left(\gamma+\varepsilon_{A}+\mu_{A}\right)\left(\gamma+\delta+\varepsilon_{C}+\mu_{C}\right) \\
& \left.+S_{C}^{d}\left(\delta+\varepsilon_{C}+\mu_{C}\right)\left(\theta_{C}\left(\gamma+\varepsilon_{A}+\mu_{A}\right)+\theta_{A} \delta \sigma\right)\right] \\
c_{1}= & \beta_{C}\left(\varepsilon_{A}+\mu_{A}\right)\left(\gamma+\delta+\varepsilon_{C}+\mu_{C}\right) \\
& \times\left[\mu_{V}\left(\gamma+\varepsilon_{A}+\mu_{A}\right)\left(\mu_{V}+\tau\right)-\beta_{A} \theta_{A} \tau S_{A}^{d}\right] \\
& +\beta_{A}\left(\delta+\varepsilon_{C}+\mu_{C}\right)\left[\mu_{V}\left(\gamma+\varepsilon_{A}+\mu_{A}\right)\left(\gamma+\delta+\varepsilon_{C}+\mu_{C}\right)\left(\mu_{V}+\tau\right)\right. \\
& \left.\quad-\beta_{C} \tau S_{C}^{d}\left(\theta_{C}\left(\gamma+\varepsilon_{A}+\mu_{A}\right)+\delta \sigma \theta_{A}\right)\right] \\
& +\left(\varepsilon_{A}+\mu_{A}\right)\left(\delta+\varepsilon_{C}+\mu_{C}\right)\left(\mu_{V}+\tau\right)\left[\beta_{A} \theta_{A} S_{A}^{d}\left(\gamma+\delta+\varepsilon_{C}+\mu_{C}\right)\right. \\
& \left.\left.+\beta_{A} \beta_{C} \theta_{A} \delta \sigma \tau S_{C}^{d}\left(\gamma+\delta+\varepsilon_{A}+\mu_{A}\right)+\delta \sigma \theta_{A}\right)\right] \\
c_{0}= & \left(\delta+\gamma+\varepsilon_{C}+\mu_{C}\right)\left(\delta+\varepsilon_{C}+\mu_{C}\right)\left(\varepsilon_{A}+\mu_{A}\right)\left(\gamma+\varepsilon_{A}+\mu_{A}\right) \mu_{V}\left(\mu_{V}+\tau\right)\left(1-R_{0}\right),
\end{aligned}
$$




$$
\begin{gathered}
S_{C}^{d}=\frac{(1-p s)\left(f_{C} Q_{C}+T\right)}{\delta+\varepsilon_{C}+\mu_{C}}, \\
S_{A}^{d}=\frac{(1-q s) f_{A} Q_{A}+\delta \sigma S_{C}^{d}}{\varepsilon_{A}+\mu_{A}}, \\
R_{0}=\frac{\tau\left[\beta_{C} S_{C}^{d}\left(\theta_{C}\left(\gamma+\varepsilon_{A}+\mu_{A}\right)+\theta_{A} \delta \sigma\right)+\beta_{A} \theta_{A} S_{A}^{d}\left(\delta+\gamma+\varepsilon_{C}+\mu_{C}\right)\right]}{\mu_{V}\left(\gamma+\varepsilon_{A}+\mu_{A}\right)\left(\delta+\gamma+\varepsilon_{C}+\mu_{C}\right)\left(\mu_{V}+\tau\right)} .
\end{gathered}
$$

The zeros of the polynomial $M+N$ determine the equilibrium of model (2.13)-(2.18). We analyze the zeros of the polynomial $M+N$ into two cases. The first case is if there is no incoming infected immigrant, so the incoming immigrants are susceptible or have permanent immunity to the dengue infection. In this case, only polynomial $M$ determines the equilibrium. The second case is if there are some incoming infected immigrants. In the second case, both polynomials $M$ and $N$ determine the equilibrium.

\subsection{No Incoming Infected Immigrants}

In this subsection, we consider the case where there is no incoming infected immigrant or mathematically $g_{C}=g_{A}=0$. Furthermore, the condition $g_{C}=g_{A}=0$ implies that polynomial $N$ becomes a zero polynomial.

In this case, model (2.13)-(2.18) has a disease-free equilibrium; that is, $E^{d}=\left(S_{C^{\prime}}^{d} 0, S_{A^{\prime}}^{d}\right.$ $0,0,0)$, where $S_{C}^{d}$ and $S_{A}^{d}$ are exactly as in (3.11)-(3.12). This equilibrium is obtained by substituting $I_{V}=0$ into (3.5).

If the vaccination programme is not implemented $(p=q=0)$ and all immigrants are susceptibles $\left(f_{C}=f_{A}=1\right)$, then we obtain $S_{C}^{d}=S_{A}^{d}=1$ and $R_{C}^{d}=R_{A}^{d}=0$. In the limiting case where all susceptible immigrants and all births are vaccinated $(p=q=1)$ and the vaccine efficacy is perfect $(s=1)$, we have $S_{C}^{d}=S_{A}^{d}=0$ and $R_{C}^{d}=R_{A}^{d}=1$.

Basic reproduction ratio is the expected number of secondary cases per primary case in a "virgin" population [19]. It is an important threshold because it determines whether an initial infection in a virgin population will end up in an endemic. This threshold parameter is given by the spectral radius of the next-generation matrix. The spectral radius of our nextgeneration matrix is the square root of $R_{0}$, where $R_{0}$ is exactly as in (3.13). This square root of $R_{0}$ can be interpreted as the basic reproduction ratio under vaccination programme.

Next, we explore the existence of the endemic equilibrium of model (2.13)-(2.18) when $g_{C}=g_{A}=0$. Here, we consider the equation $c_{2} I_{V}^{2}+c_{1} I_{V}+c_{0}=0$, where the coefficients $c_{0}, c_{1}$, and $c_{2}$ are as in (3.8)-(3.10).

It can be seen that $c_{2}$ is positive. The coefficient $c_{0}$ is positive for $R_{0}<1$, and it is negative for $R_{0}>1$. Moreover, for $R_{0}=1$, we have that $c_{0}=0$ and $c_{1}, c_{2}>0$. So, model (2.13)-(2.18) cannot exhibit backward bifurcation at $R_{0}=1$.

For $R_{0} \leq 1$, we have following inequalities:

$$
\begin{gathered}
\beta_{C} \tau S_{C}^{d}\left(\theta_{C}\left(\gamma+\varepsilon_{A}+\mu_{A}\right)+\delta \sigma \theta_{A}\right)<\mu_{V}\left(\gamma+\varepsilon_{A}+\mu_{A}\right) \\
\left(\delta+\gamma+\varepsilon_{C}+\mu_{C}\right)\left(\mu_{V}+\tau\right) \beta_{A} \theta_{A} \tau S_{A}^{d} \\
<\mu_{V}\left(\gamma+\varepsilon_{A}+\mu_{A}\right)\left(\mu_{V}+\tau\right) .
\end{gathered}
$$




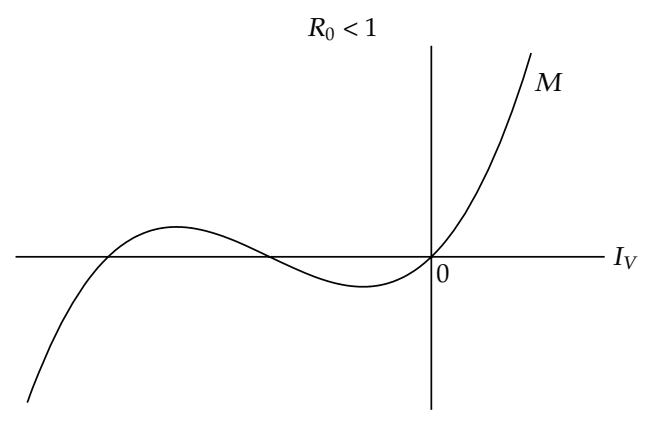

(a)

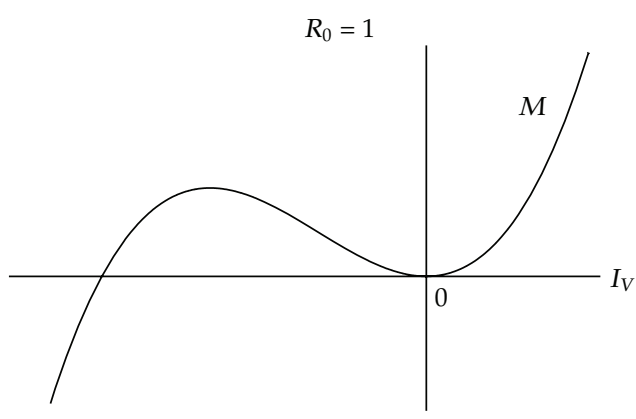

(b)

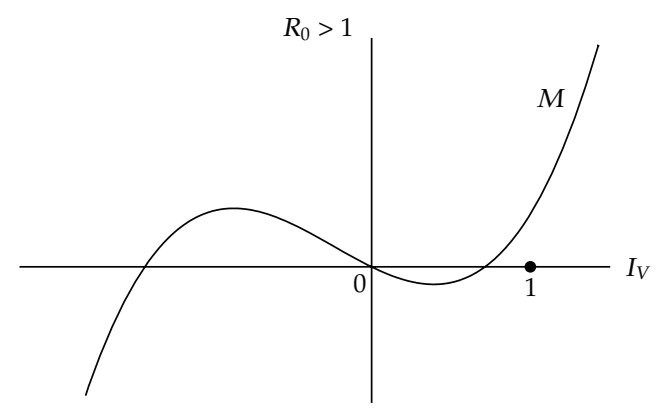

(c)

Figure 2: Three typical graphs of the polynomial $M$ in (3.6) with respect to three conditions of $R_{0}$.

These inequalities imply $c_{1}>0$ for $R_{0} \leq 1$. Thus, there is no positive root $I_{V}$ of the equation $c_{2} I_{V}^{2}+c_{1} I_{V}+c_{0}=0$ for $R_{0} \leq 1$. And there is a unique positive root $I_{V}^{e}$ of the equation $c_{2} I_{V}^{2}+c_{1} I_{V}+c_{0}=0$ which is always less than one for $R_{0}>1$. Figure 2 gives three qualitative graphs of $M$ with respect to the three conditions of $R_{0}$.

It can be verified that the equilibrium $E^{e}=\left(S_{C}^{e}, I_{C}^{e}, S_{A}^{e}, I_{A}^{e}, E_{V}^{e}, I_{V}^{e}\right)$ whose coordinates satisfy equations (3.5) is $\operatorname{in} \operatorname{int}(\Omega)$ if and only if $R_{0}>1$. We summarized these results in the following proposition.

Proposition 3.1. Let $g_{C}=g_{A}=0$. Model (2.13)-(2.18) always has a unique disease-free equilibrium $E^{d}$ in $\Omega$. For $R_{0}>1$, model (2.13)-(2.18) also has a unique positive endemic equilibrium $E^{e}$ in int $(\Omega)$ whose components satisfy (3.5), and $I_{V}$ satisfies $c_{2} I_{V}^{2}+c_{1} I_{V}+c_{0}=0$, where the coefficients $c_{0}, c_{1}, c_{2}$ are as in (3.8)-(3.10).

The next proposition gives the stability of equilibrium $E^{d}$.

Proposition 3.2. Let $g_{C}=g_{A}=0$. The disease-free equilibrium $E^{d}$ is locally asymptotically stable if $R_{0}<1$ and it is unstable if $R_{0}>1$.

Proof. The linearization of model (2.13)-(2.18) at point $E^{d}$ gives the Jacobian matrix:

$$
A=\left(\begin{array}{cc}
A_{1} & A_{3} \\
0 & A_{2}
\end{array}\right),
$$


where

$$
\begin{aligned}
A_{1} & =\left(\begin{array}{cc}
-\delta-\varepsilon_{C}-\mu_{C} & 0 \\
\delta \sigma & -\varepsilon_{A}-\mu_{A}
\end{array}\right), \\
A_{2} & =\left(\begin{array}{cccc}
-\delta-\gamma-\varepsilon_{C}-\mu_{C} & 0 & 0 & \beta_{C} S_{C}^{d} \\
\delta \sigma & -\gamma-\varepsilon_{A}-\mu_{A} & 0 & \beta_{A} S_{A}^{d} \\
\theta_{C} & \theta_{A} & -\mu_{V}-\tau & 0 \\
0 & 0 & \tau & -\mu_{V}
\end{array}\right) .
\end{aligned}
$$

Moreover, the eigenvalues of matrices $A_{1}$ and $A_{2}$ determine the local stability of $E^{d}$.

The eigenvalues of matrix $A_{1}$ are $-\left(\delta+\varepsilon_{C}+\mu_{C}\right)$ and $-\left(\varepsilon_{A}+\mu_{A}\right)$. The matrix $-A_{2}$ is an Mmatrix. The real parts of all eigenvalues of matrix $-A_{2}$ are positive if and only if $\operatorname{det}\left(-A_{2}\right)>0$ (see [20]). Furthermore, all eigenvalues of $A_{2}$ have negative real parts if and only if $\operatorname{det}\left(A_{2}\right)>$ 0 . The determinant of matrix $A_{2}$ is given by

$$
\operatorname{det}\left(A_{2}\right)=-\mu_{V}\left(\gamma+\varepsilon_{A}+\mu_{A}\right)\left(\delta+\gamma+\varepsilon_{C}+\mu_{C}\right)\left(\mu_{V}+\tau\right)\left(R_{0}-1\right) .
$$

Thus, if $R_{0}<1$, then the equilibrium $E^{d}$ is locally asymptotically stable and it is unstable if $R_{0}>1$.

Let the endemic equilibrium $E^{e}=\left(S_{C}^{e}, I_{C^{\prime}}^{e} S_{A^{\prime}}^{e} I_{A^{\prime}}^{e} E_{V}^{e}, I_{V}^{e}\right)$ exists. Linearization of model (2.13)-(2.18) at point $E^{e}$ gives following Jacobian matrix:

$$
J=\left(\begin{array}{cccccc}
\Delta_{1} & 0 & 0 & 0 & 0 & -\beta_{C} S_{C}^{e} \\
\beta_{C} I_{V}^{e} & \Delta_{2} & 0 & 0 & 0 & \beta_{C} S_{C}^{e} \\
\delta \sigma & 0 & \Delta_{3} & 0 & 0 & -\beta_{A} S_{A}^{e} \\
0 & \delta \sigma & \beta_{A} I_{V}^{e} & \Delta_{4} & 0 & \beta_{A} S_{A}^{e} \\
0 & S_{V}^{e} \theta_{C} & 0 & \theta_{A} S_{V}^{e} & \Delta_{5} & -\left(\theta_{A} I_{A}^{e}+\theta_{C} I_{C}^{e}\right) \\
0 & 0 & 0 & 0 & \tau & -\mu_{V}
\end{array}\right),
$$

where $\Delta_{1}=-\left(\beta_{C} I_{V}^{e}+\delta+\varepsilon_{C}+\mu_{C}\right), \Delta_{2}=-\left(\gamma+\delta+\varepsilon_{C}+\mu_{C}\right), \Delta_{3}=-\left(\beta_{A} I_{V}^{e}+\varepsilon_{A}+\mu_{A}\right), \Delta_{4}=$ $-\left(\gamma+\varepsilon_{A}+\mu_{A}\right), \Delta_{5}=-\left(\theta_{A} I_{A}^{e}+\theta_{C} I_{C}^{e}+\mu_{V}+\tau\right)$, and $S_{V}^{e}=1-E_{V}^{e}-I_{V}^{e}$.

It is not easy to prove analytically that all eigenvalues of $J$ have negative real parts for $R_{0}>1$. However, from our numerical simulations (case $R_{0}>1$ ) all of the eigenvalues have negative real parts. Figure 3 gives the projection of three orbits of three different initial conditions when $R_{0}>1$ on the $I_{C}-I_{A}$ plane. The component $\left(I_{C}, I_{A}\right)$ of the equilibrium $E^{e}$ is 


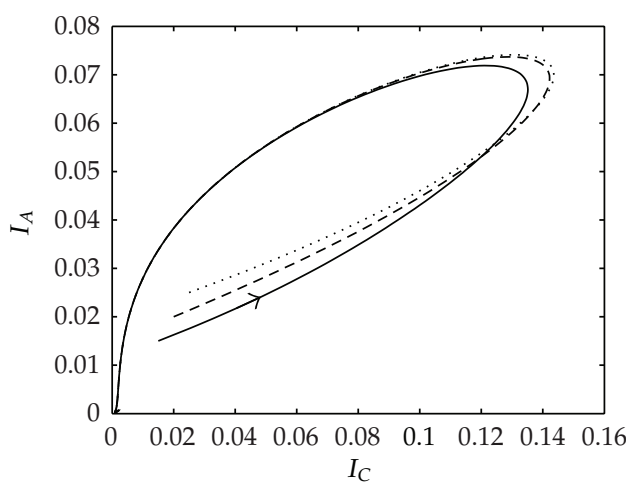

(a)

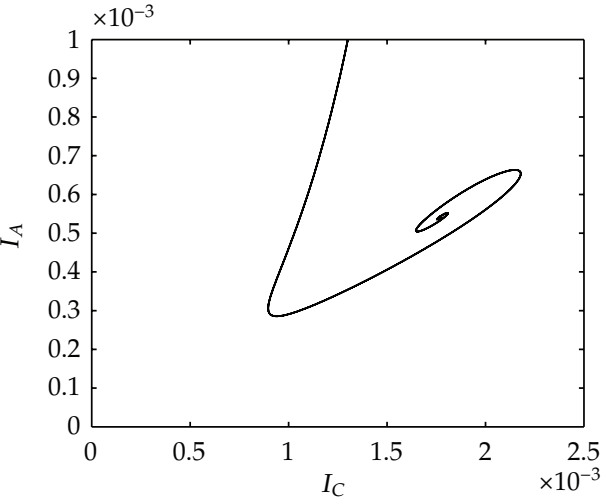

(b)

Figure 3: Projection of three orbits of model (2.13)-(2.18) on $I_{C}-I_{A}$ plane (a) and the projection near the equilibrium state (b). This projection indicates the local stability of the endemic equilibrium $E^{e}$ when $R_{0}>1$.

not $(0,0)$. This simulation indicates that the endemic equilibrium $E^{e}$ is locally asymptotically stable when $R_{0}>1$.

\subsection{Some Incoming Immigrants Are Infected}

Here, we consider the case that there are some infected incoming immigrants; that is, $g_{C}$ or $g_{A}$ is larger than zero. In this case, we have following proposition.

Proposition 3.3. Let $g_{C}$ or $g_{A}$ be larger than zero. Model (2.13)-(2.18) always has a unique positive endemic equilibrium $E^{f}$ in $\operatorname{int}(\Omega)$ whose components satisfy (3.5) and $I_{V}$ satisfies $M\left(I_{V}\right)+N\left(I_{V}\right)=0$.

We will give the outline of proof of Proposition 3.3.

Outline of proof. When $g_{C}$ or $g_{A}$ or both are larger than zero, the cubic polynomial $N$ in (3.7) always has two negative zeros and one positive zero which is less than one. Figure 4 gives the graph of the polynomial $N$.

The cubic polynomial $M$ in (3.6) always has a trivial zero. Depending on $R_{0}$, the other two zeros could be negative, zero, or positive. Figure 2 illustrates three typical graphs of the polynomial $M$ with respects to $R_{0}$.

Figure 5 gives the graph of polynomial $M+N$. The graph always has two negative zeros and one positive zero which is less than one. This positive zero is the component $I_{V}$ of endemic equilibrium $E^{f}$.

From Proposition 3.3, there is no disease-free equilibrium and there is only endemic equilibrium if there are always some infected incoming child or adult immigrants. So, it is very important to do screening for the child and adult immigrants. The infected immigrants should be quarantined as long as they are ill. Otherwise, we will lose the disease-free condition. Here, we get a similar conclusion as in [14]. In [14], the authors did not separate the child class and the adult class in their model.

Figures 6 and 7 show the values of the equilibrium infected child population $I_{C}$ and the equilibrium infected adult population $I_{A}$ as the function of the portion of infected child 


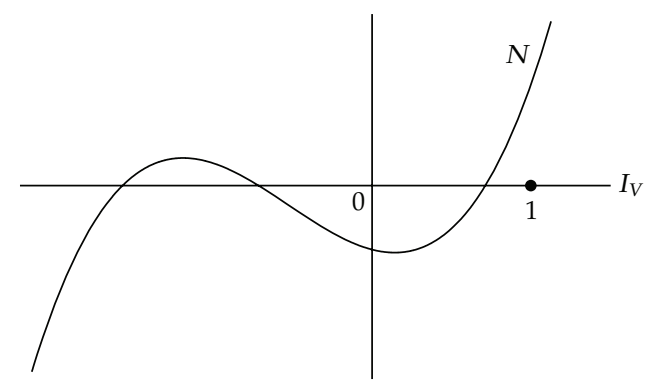

Figure 4: Graph of the polynomial N.

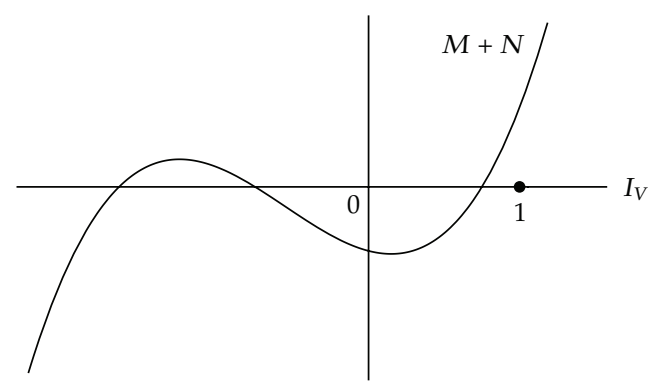

Figure 5: Graph of the polynomial $M+N$.

$g_{C}$ and adult immigrants $g_{A}$. In Figure 6, we use parameters which produce $R_{0}<1$ around $g_{C}=g_{A}=0$. Note that the lowest point $\left(g_{C}=g_{A}=0\right)$ corresponds to the components $I_{C}^{d}$ and $I_{A}^{d}$ of the disease-free equilibrium $E^{d}$. When $g_{C}, g_{A} \neq 0$, the points in the surface correspond to the components $I_{C}^{f}$ and $I_{A}^{f}$ of the endemic equilibrium $E^{f}$. However, in Figure 7, we use parameters which produce $R_{0}>1$ around $g_{C}=g_{A}=0$. Here, the lowest point $\left(g_{C}=g_{A}=0\right)$ corresponds to the components $I_{C}^{e}$ and $I_{A}^{e}$ of the endemic equilibrium $E^{e}$. When $g_{C}, g_{A} \neq 0$, the points in the surface correspond to the components $I_{C}^{f}$ and $I_{A}^{f}$ of endemic equilibrium $E^{f}$. Despite the difference in the resulting properties of the basic reproduction number, and since both $I_{A}$ and $I_{C}$ constitute the endemic equilibrium $E_{f}$, the figures in fact indicate the existence of this endemic equilibrium when $g_{C}$ and $g_{A}$ are not zero.

The stability of the endemic equilibrium $E^{f}$ is not easy to be obtained analytically. Numerical simulations indicate the local stability of the equilibrium $E^{f}$. Figure 8 gives three orbits of three different sets of parameter values. This simulation indicates that the equilibrium $E^{f}$ is locally asymptotically stable.

\section{Numerical Simulation}

In the following numerical simulations, we use data in Table 1.

In Figure 9, we simulate four different scenarios, relative to no vaccination scenario and low screening level, that is, $g_{a}=g_{c}=20 \%$. The situation is described as follow, first if we raise the level of screening twice, that is, reduction of $g_{a}$ and $g_{c}$ from $20 \%$ to $10 \%$, the infection will decrease from $100 \%$ to $85.7 \%$ for $I_{a}$ and $94 \%$ for $I_{c}$. If we gain the screening process up to four times, we have the infection decreasing from $100 \%$ to $82.1 \%$ for $I_{a}$ and $91.5 \%$ for $I_{c}$. 


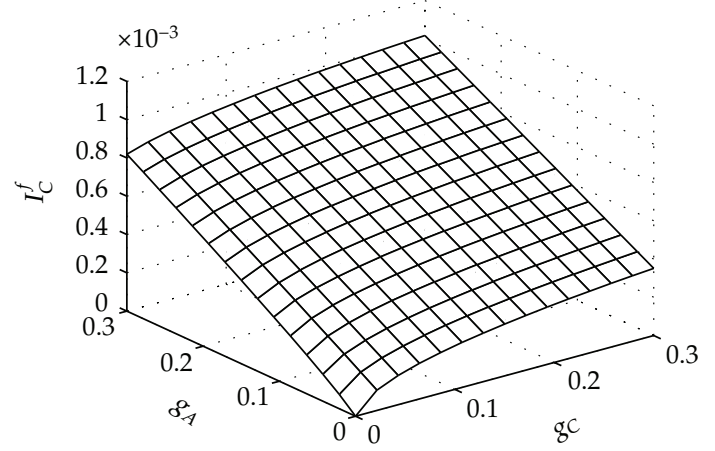

(a)

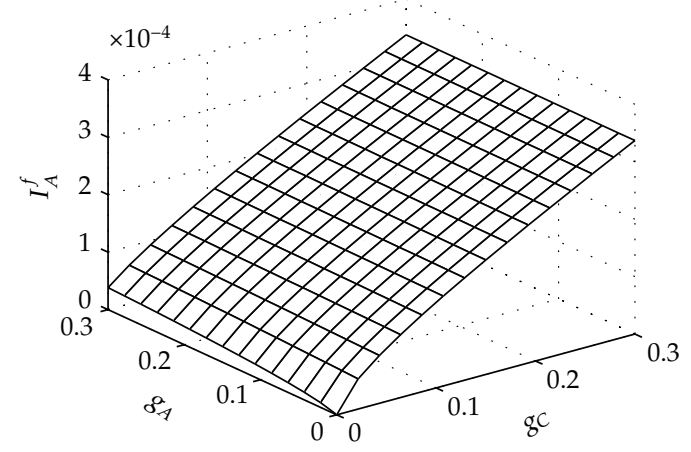

(b)

Figure 6: Component $I_{C}(\mathrm{a})$ and component $I_{A}(\mathrm{~b})$ of endemic equilibria $E^{f}$. Here, $R_{0}<1$ when $g_{C}=g_{A}=0$.

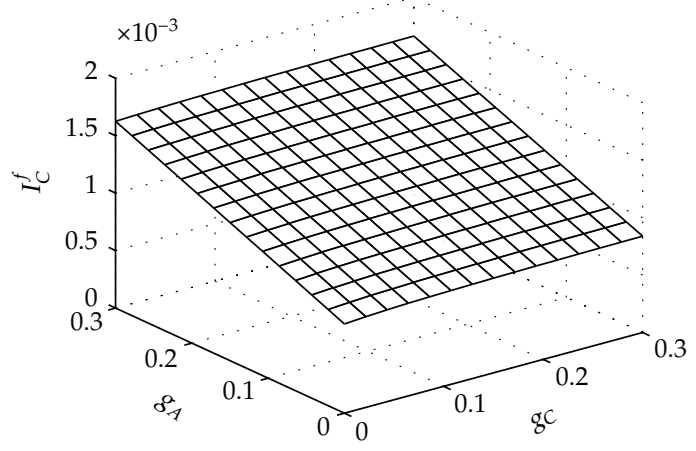

(a)

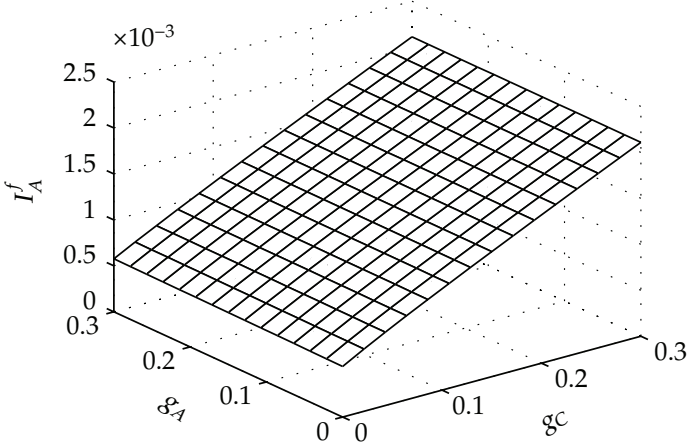

(b)

Figure 7: Component $I_{C}(\mathrm{a})$ and component $I_{A}(\mathrm{~b})$ of endemic equilibria $E^{f}$. Here, $R_{0}>1$ when $g_{C}=g_{A}=0$.

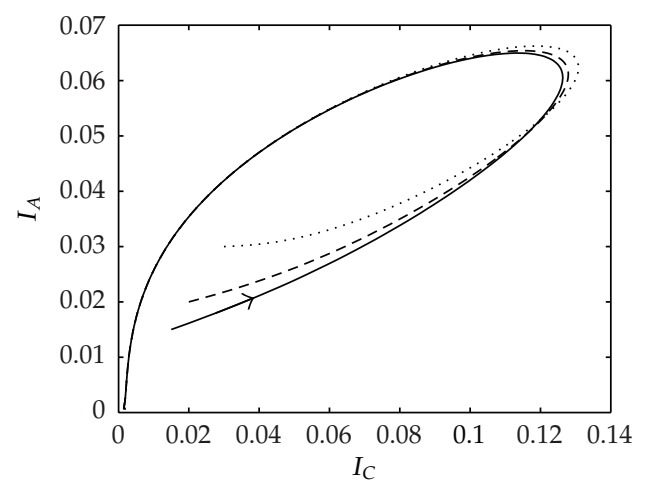

(a)

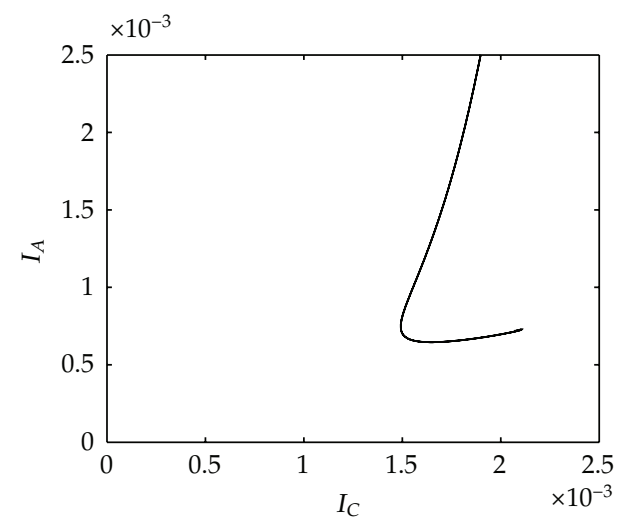

(b)

Figure 8: Projection of three orbits of model (2.13)-(2.18) on $I_{C}-I_{A}$ plane (a) and the projection near the equilibrium state (b). This projection indicates the local stability of the endemic equilibrium $E^{f}$. 
Table 1: Data for numerical simulations (y represents years).

\begin{tabular}{lccccc}
\hline Par. & Value & Par. & Value & Par. & Value \\
\hline$P_{C}$ & $200 / \mathrm{y}$ & $P_{A}$ & $2000 / \mathrm{y}$ & $P_{V}$ & $5000 / \mathrm{y}$ \\
$\lambda_{C}$ & $1168 / \mathrm{y}$ & $\lambda_{A}$ & $584 / \mathrm{y}$ & $\lambda_{V}$ & $2190 / \mathrm{y}$ \\
$1 / \mu_{C}$ & $14.8 \mathrm{y}$ & $1 / \mu_{A}$ & $60 \mathrm{y}$ & $1 / \mu_{V}$ & $1 / 12 \mathrm{y}$ \\
$f_{C}$ & 0.75 & $f_{A}$ & 0.75 & $1 / \tau$ & $1 / 24 \mathrm{y}$ \\
$g_{C}$ & 0.05 & $g_{A}$ & 0.05 & $\mathrm{~B}$ & $200 / \mathrm{y}$ \\
$\varepsilon_{C}$ & $0.2 / \mathrm{y}$ & $\varepsilon_{A}$ & $0.1 / \mathrm{y}$ & $\gamma$ & $36.5 / \mathrm{y}$ \\
$s$ & 0.95 & $1 / \delta$ & $15 \mathrm{y}$ & - & - \\
$p$ & 0.9 & $q$ & 0.9 & - & - \\
\hline
\end{tabular}

Table 2: Percentages of endemicity. We use the first scenario as a reference scenario for the other four scenarios.

\begin{tabular}{lcccc}
\hline Scenarios & $I_{A}$ & $I_{C}$ & Percentage of $I_{A}$ & Percentage of $I_{C}$ \\
\hline $\begin{array}{l}\text { No vaccination, } \\
g_{a}=g_{c}=20 \%\end{array}$ & 0.0028 & 0.0083 & $100.0 \%$ & $100.0 \%$ \\
$\begin{array}{l}\text { No vaccination, } \\
g_{a}=g_{c}=10 \%\end{array}$ & 0.0024 & 0.0078 & $85.7 \%$ & $94 \%$ \\
$\begin{array}{l}\text { No vaccination, } \\
g_{a}=g_{c}=5 \%\end{array}$ & 0.0023 & 0.0076 & $82.1 \%$ & $91.5 \%$ \\
$\begin{array}{l}\text { Vac. } p=q=40 \%, \\
g_{a}=g_{c}=20 \%\end{array}$ & 0.002 & 0.0055 & $71.4 \%$ & $66.3 \%$ \\
$\begin{array}{l}\text { Vac. } p=q=80 \%, \\
g_{a}=g_{c}=20 \%\end{array}$ & 0.0012 & 0.0027 & $42.9 \%$ & $32.5 \%$ \\
\hline
\end{tabular}

Hence, increasing the level of screening will decrease the endemicity. But if we vaccine $40 \%$ of children and adult ( $p=q=40 \%$ ), the decreasing level of infection is $71.4 \%$ for $I_{a}$ and $66.3 \%$ for $I_{c}$, and if we raise the coverage of vaccination to $80 \%(p=q=80 \%)$, we can reduce the infection up to $42.9 \%$ for $I_{a}$ and $32.5 \%$ for $I_{c}$. So, increasing the coverage of vaccination will also decrease the endemicity. The summary of the scenarios can be seen in Table 2 .

\section{Conclusion}

In this paper we derive a mathematical model of dengue transmission with vaccination program. The model incorporates two-age classes and migration. We also consider a susceptibility distribution in the incoming migrants.

From the analysis of the model, we obtain a conclusion that the susceptibility distribution is an important factor for the existence of disease-free equilibrium. If there is no incoming infected immigrant, then we have a unique disease-free equilibrium and a unique endemic equilibrium which depend on the basic reproduction ratio. Moreover, the stability of the equilibria also depends on the basic reproduction ratio. However, if some of the incoming immigrants are infected, then we only have a unique endemic equilibrium. Hence, screening for the incoming immigrants must be done. The incoming infected immigrants should be 


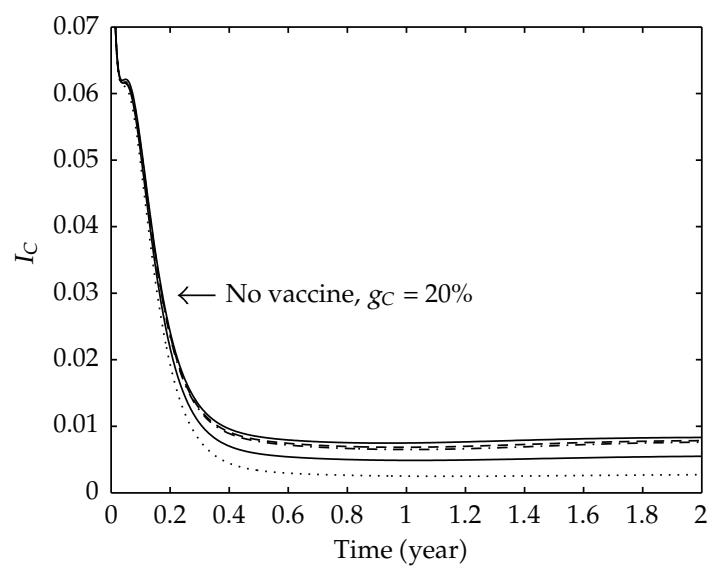

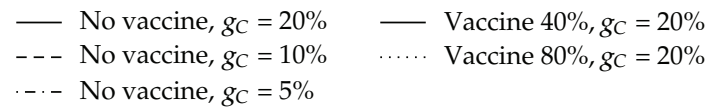

(a)

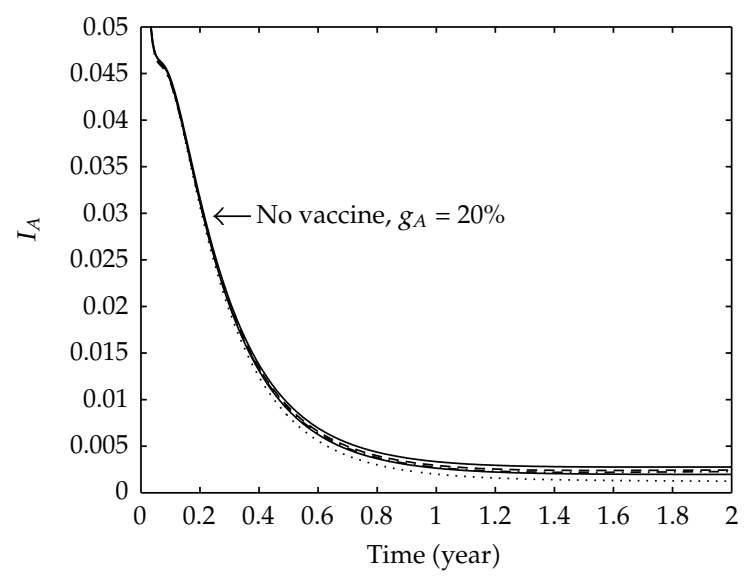

$\begin{array}{ll}\text { - No vaccine, } g_{A}=20 \% & \text { - Vaccine } 40 \%, g_{A}=20 \% \\ \text { - } & \text { No vaccine, } g_{A}=10 \% \\ \text {-. N N vaccine, } g_{A}=5 \% & \end{array}$

(b)

Figure 9: Dynamics of some scenarios. Here, we take $(0.3,0.1,0.5,0.1,0.1,0.1)$ as the initial condition.

quarantined until they are recovered. Otherwise, we will lose the disease-free state from the population.

From the sensitivity analysis of the level of screening and the coverage of vaccination, increasing one of these parameters will give the reduction of endemic level. Increasing both parameters will give larger reduction of endemic level. The resulting simulation could give prior information for policy maker in setting the scale of vaccination and understanding the effect of vaccination in the reduction of endemic level. 


\section{Acknowledgment}

Part of the research is funded by Hibah Pasca Sarjana of the Directorate for Higher Education.

\section{References}

[1] S. Busenberg, K. Cooke, and M. Iannelli, "Endemic thresholds and stability in a class of age-structured epidemics," SIAM Journal on Applied Mathematics, vol. 48, no. 6, pp. 1379-1395, 1988.

[2] D. S. Shepard, J. A. Suaya, S. B. Halstead et al., "Cost-effectiveness of a pediatric dengue vaccine," Vaccine, vol. 22, no. 9-10, pp. 1275-1280, 2004.

[3] J. Müller, "Optimal vaccination patterns in age-structured populations: endemic case," Mathematical and Computer Modelling, vol. 31, no. 4-5, pp. 149-160, 2000.

[4] D. Greenhalgh, "Threshold and stability results for an epidemic model with an age-structured meeting rate," IMA Journal of Mathematics Applied in Medicine and Biology, vol. 5, no. 2, pp. 81-100, 1988.

[5] H. Inaba, "Threshold and stability results for an age-structured epidemic model," Journal of Mathematical Biology, vol. 28, no. 4, pp. 411-434, 1990.

[6] E. Shim, Z. Feng, M. Martcheva, and C. Castillo-Chavez, "An age-structured epidemic model of rotavirus with vaccination," Tech. Rep. 2, Statistical and Applied Mathematical Sciences Institute, 2006.

[7] P. Pongsumpun, Y. Lenbury, and I. M. Tang, "Age structure in a model for the transmission of dengue haemorrhagic fever in Thailand," East-West Journal of Mathematics, pp. 93-103, 2002.

[8] P. Pongsumpun and I. M. Tang, "Transmission of Dengue hemorrhagic fever in an age structured population," Mathematical and Computer Modelling, vol. 37, no. 9-10, pp. 949-961, 2003.

[9] A. K. Supriatna, E. Soewono, and S. A. van Gils, "A two-age-classes dengue transmission model," Mathematical Biosciences, vol. 216, no. 1, pp. 114-121, 2008.

[10] L. Esteva and C. Vargas, "Analysis of a dengue disease transmission model," Mathematical Biosciences, vol. 150, no. 2, pp. 131-151, 1998.

[11] K. R. Porter, C. G. Beckett, H. Kosasih et al., "Epidemiology of dengue and dengue hemorrhagic fever in a cohort of adults living in Bandung, West Java, Indonesia," American Journal of Tropical Medicine and Hygiene, vol. 72, no. 1, pp. 60-66, 2005.

[12] D. Chemtob and Z. Grossman, "Epidemiology of adult and adolescent HIV infection in Israel: a country of immigration," International Journal of STD and AIDS, vol. 15, no. 10, pp. 691-696, 2004.

[13] L. R. Krilov, "Emerging infectious disease issues in international adoptions: severe acute respiratory syndrome (SARS), avian influenza and measles," Current Opinion in Infectious Diseases, vol. 17, no. 5, pp. 391-395, 2004.

[14] F. Brauer and P. van den Driessche, "Models for transmission of disease with immigration of infectives," Mathematical Biosciences, vol. 171, no. 2, pp. 143-154, 2001.

[15] G. Chowell, P. W. Fenimore, M. A. Castillo-Garsow, and C. Castillo-Chavez, "SARS outbreaks in Ontario, Hong Kong and Singapore: the role of diagnosis and isolation as a control mechanism," Journal of Theoretical Biology, vol. 224, no. 1, pp. 1-8, 2003.

[16] The Pan American Health Organization, Report of the Workshop on Dengue Burden Studies, 2002.

[17] C. Castillo-Chavez and H. R. Thieme, "Asymptotically autonomous epidemic models," in Mathematical Population Dynamics: Analysis of Heterogeneity, vol. 1 of Theory of Epidemics, pp. 33-50, Wuerz, 1995.

[18] H. R. Thieme, "Asymptotically autonomous differential equations in the plane," The Rocky Mountain Journal of Mathematics, vol. 24, no. 1, pp. 351-380, 1994.

[19] O. Diekmann and J. A. P. Heesterbeek, Mathematical Epidemiology of Infectious Diseases, John Wiley \& Sons, Chichester, UK, 2000.

[20] A. Berman and R. J. Plemmons, Nonnegative Matrices in the Mathematical Sciences, vol. 9, Society for Industrial and Applied Mathematics SIAM, Philadelphia, Pa, USA, 1994. 


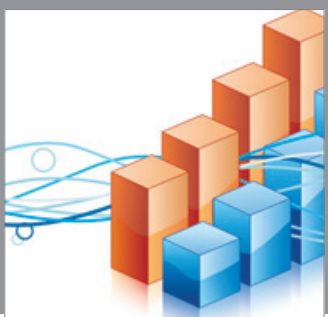

Advances in

Operations Research

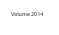

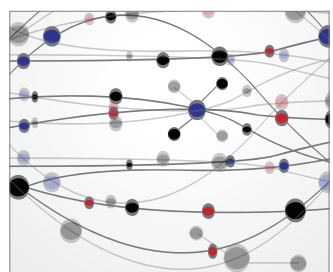

\section{The Scientific} World Journal
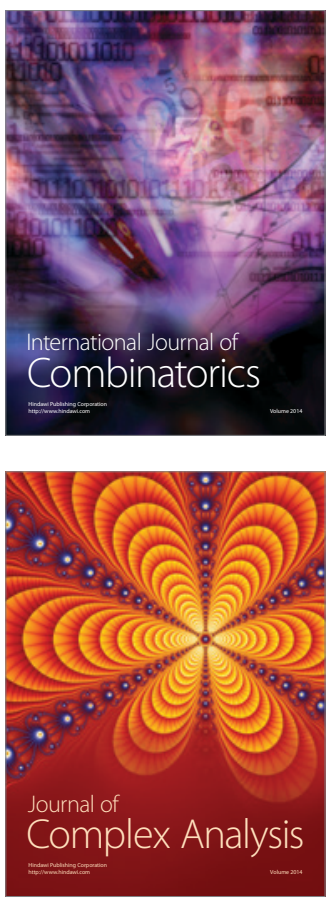

International Journal of

Mathematics and

Mathematical

Sciences
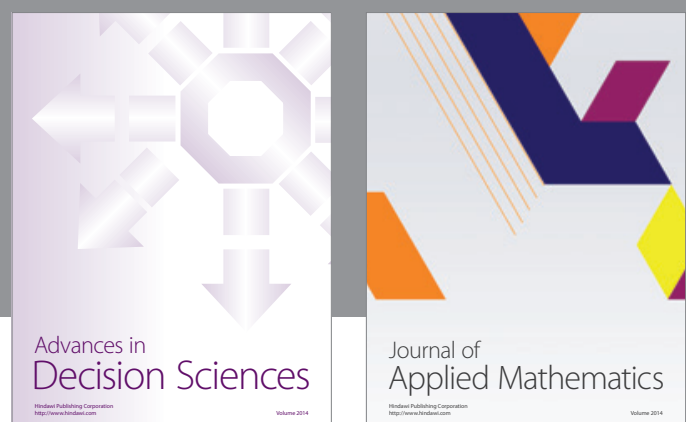

Journal of

Applied Mathematics
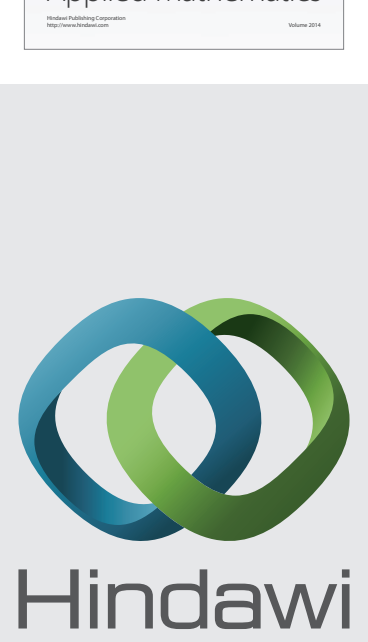

Submit your manuscripts at http://www.hindawi.com
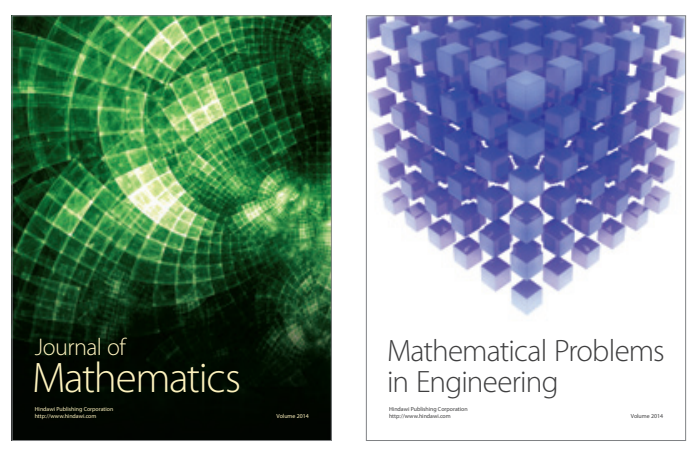

Mathematical Problems in Engineering
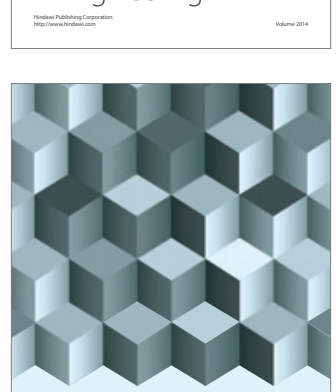

Journal of

Function Spaces
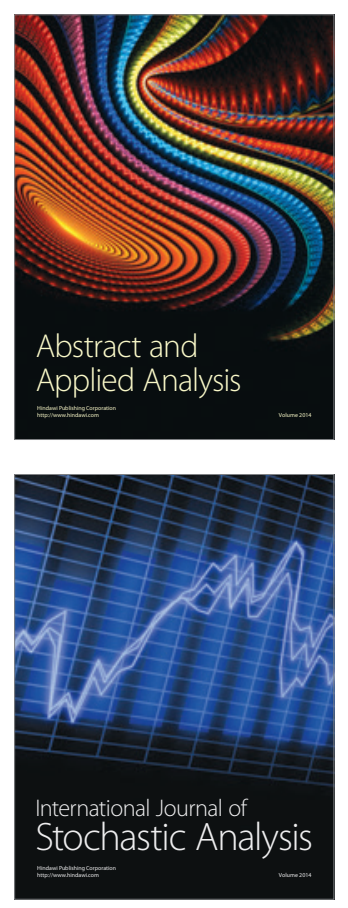

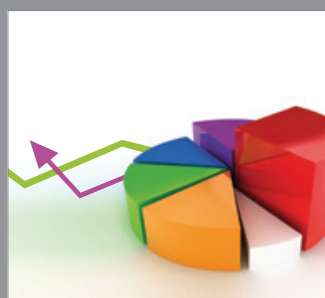

ournal of

Probability and Statistics

Promensencen
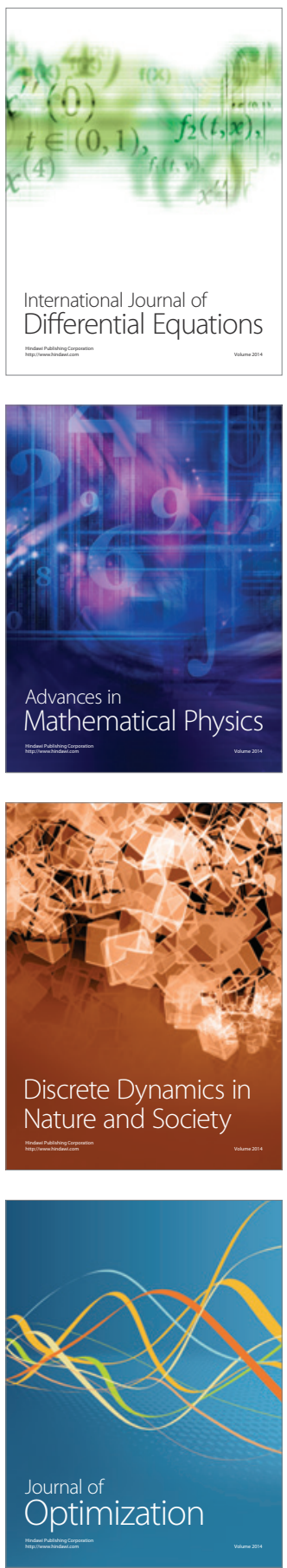